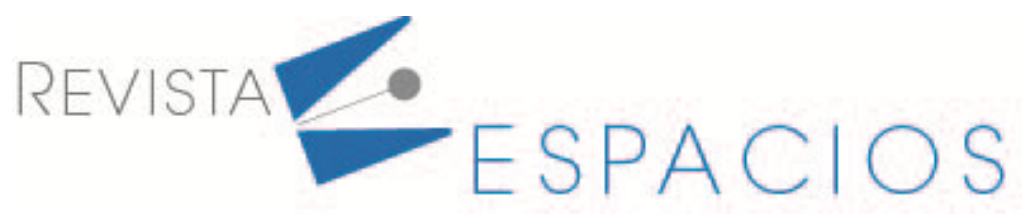

\title{
Laboratorios Remotos e IOT una oportunidad para la formación en ciencias e ingeniería en tiempos del COVID- 19: Caso de Estudio en Ingeniería de Control
}

\section{Remote Laboratories an opportunity for formation in science and engineering in times of SARS- COV-2: Case Study in Control Engineering.}

\author{
VARGAS, Javier ${ }^{1}$ \\ CUERO, Jairo ${ }^{2}$ \\ TORRES, Camilo ${ }^{3}$
}

\section{Resumen}

Este artículo presenta los resultados del uso de laboratorios remotos aplicados a la formación elearning en ingeniería y ciencias básicas en tiempos del COVID-19. La investigación se llevó acabo porque el coronavirus afectó el sector educación especialmente en la formación de las áreas experimentales donde requieren manipular variables físicas. Los resultados evidenciaron que los estudiantes percibieron una sensación de inmersión en un laboratorio real a pesar de que la experimentación fue mediante la web.

Palabras claves: COVID -19, educación en ingeniería, educación en tiempos de pandemia, laboratorios remoto para educación, internet de las cosas, tecnologías emergentes.

\begin{abstract}
This article presents the results of the use of remote laboratories applied to e-learning training in engineering and basic sciences in times of COVID-19. The investigation was motivated by the impact that the coronavirus had on the education sector, especially in the formation of experimental areas where a practical component is mandatory for manipulating physical variables. Students perceived a feeling of immersion like in a real environment despite being web-based experimentation.

key words: COVID -19 , engineering education, education in times of pandemic, remote laboratories for education, internet of things, emerging technologies
\end{abstract}

\section{Introducción}

La situación mundial generada por el COVID-19 ha ocasionado que diversas instituciones y académicos recomienden la apropiación del e-learning como medida de contención para la continuidad de la educación que

\footnotetext{
${ }^{1}$ Doctor En ciencias de la Educación, Universidad Metropolitana de Educación Ciencia y Tecnología (UMECIT -Panamá)- Investigador del grupo Macrypt y del grupo EYSI, de la Universidad de los Llanos. Javier.andres.vargas@unillanos.edu.co

${ }^{2}$ Candidato a Magister en Automatización, Universidad Nacional de Colombia - Investigador del grupo Macrypt y del grupo EYSI, de la Universidad de los Llanos, Jairo_cuero@unilllanos.edu.co

${ }^{3}$ Candidato a Doctor en Educación, Universidad Católica de Manizales, Investigador del grupo Macrypt, de la Universidad de los. friveros@unillanos.edu.co
} 
ahora debe sumar a sus retos las implicaciones del aislamiento. No solo aparece como desafío lograr la reducción de las brechas digitales existentes que dificultan el acceso a las clases virtuales, sino que se deben implementar prácticas para disminuir la deserción, por ejemplo, a través de la formación integral, la motivación pedagógica y el cambio hacia metodologías que integren el uso de herramientas tecnológicas, como las utilizadas en la educación virtual y a distancia (Gazzo, 2020, Oliva, 2020).

Según el informe La educación en tiempos de la pandemia de COVID-19 (CEPAL 2020), 29 países de América Latina y del Caribe continuaron con el proceso educativo a través de distintas modalidades como virtual, a distancia, mixta, con encuentros sincrónicos y asincrónicos, en todos los niveles de formación. La educación virtual ha sido sin duda la gran protagonista por estos tiempos debido a que ha ayudado a reducir las barreras de la distancia en la enseñanza, brindando flexibilidad respecto a los tiempos de estudio, generando contenido digital que aporta al proceso de aprendizaje adaptativo de los estudiantes.

Asimismo, el aumento de las herramientas virtuales favorece el cambio del medio presencial al virtual en la educación transversal y multidisciplinaria que incluye diversas áreas o ámbitos y que a través de una organización flexible responde a las necesidades formativas de los estudiantes y los acerca a las herramientas del mundo profesional al que se enfrentarán en un futuro (Sangrá, 2001). No obstante, la adecuada planificación de las actividades y la combinación de metodologías que apoyen las interacciones colectivas continúa siendo crucial (Moreira-Segura \& Delgadillo-Espinoza, 2015).

De igual manera, la educación a distancia se ha fortalecido en los últimos años debido a las ventajas que ofrece como son el ahorro en costos de infraestructura física, la reducción en los tiempos de desplazamiento, la accesibilidad y el desarrollo de las competencias transversales como el uso de las TIC. Sin embargo, en los estudios de esta alternativa se han afrontado múltiples obstáculos y desafíos entre ellos la formación en experimentación, especialmente en áreas como las ciencias y las ingenierías donde la práctica hace parte significativa del proceso de aprendizaje (Calvo et al., 2009). Para superar esto se presentan principalmente dos alternativas, los Laboratorios Virtuales (LV) o los Laboratorios Remotos (LR), en el primer caso, los procesos y prácticas se desarrollan en simuladores que intentan recrear las condiciones reales del entorno, en el segundo, el estudiante realiza las prácticas en un laboratorio real localizado en un lugar remoto y lo manipula a distancia a través de internet. Estas alternativas se han utilizado cuando existen dificultades para la asistencia a las aulas físicas (Zaldívar Colado, 2019) o cuando el tiempo requerido para realizar las actividades en el laboratorio supera el asignado, o como forma complementaria a las prácticas presenciales (Masanet, et al., 2020)

Los laboratorios remotos, además de contar con un sistema computacional como los laboratorios virtuales, se basan en una arquitectura web cliente - servidor pero además, poseen toda la instrumentación, control y acceso a equipos reales lo que permite el aprovechamiento de laboratorios convencionales (LC) existentes (Zamora Musa, R., 2010). Dentro de las características más importantes de los laboratorios remotos está la posibilidad de operar a distancia un equipo real con todas las perturbaciones que el entorno pueda tener, lo que permite enriquecer el proceso de aprendizaje y la consolidación de conceptos a través de la observación, interpretación, comparación y análisis de los resultados (Canu \& Duque, 2015), igualmente, los LR cuentan con esquemas de seguridad que impiden el daño de equipos y puesto que son operados de manera remota no representan ningún riesgo para los usuarios, por otro lado, dan la libertad de experimentar a un ritmo propio, con pocas restricciones de horario y sin la necesidad de desplazarse hasta las instalaciones del laboratorio (Medina et al., 2011).

En 1989, William Wulf acuñaba el término colaboratorio para describir un centro sin paredes en el que se realizan investigaciones sin importar la ubicación física y se tiene acceso a instrumentos, datos, recursos computacionales e información de otros investigadores. De igual manera, Kouzes et al., (1996) vaticinaban que en estos centros proliferaría la telepresencia y los laboratorios virtuales, y que los educadores debían adoptar nuevas estrategias, también hacían referencia al trabajo en espacios ciber físicos. 
Desde entonces han aparecido diversas aplicaciones de laboratorios remotos, la mayoría de ellas en ingeniería y física. Para el 2004 se encontraban 70 LR y para el 2006120 LR (Matarrita \& Concari, 2015), estos proyectos se desarrollaron entre varias entidades y con equipos interdisciplinarios principalmente conformados por universidades para promover el aprendizaje y como una posibilidad para desarrollar estrategias docentes.

En electrónica análoga se destacan laboratorios como VISIR (Tawfik et al., 2012), NetLab (Nedic, Z., \& Machotka, J. F.,2007) y LaboREM (Luthon, F., \& Larroque, B. ,2014) para el desarrollo de prácticas con circuitos eléctricos usando componentes pasivos y amplificadores operacionales para el diseño y análisis de osciladores y filtros. Con éstos tres proyectos se desarrolló un sistema de evaluación formativa principalmente en la plataforma VISIR que permite el análisis automático de la experiencia (Loro, 2018). A su vez, en el campo de las energías renovables está una herramienta educativa para los estudiantes en los cursos de ingeniería en turbomáquinas que permite la determinación de pérdidas de perfil a través de una fila de palas de turbina de baja presión en condiciones de flujo subsónico bajo (Monaco et al., 2013), igualmente se presenta una tesis doctoral que desarrolló un laboratorio remoto para la medición de la eficiencia energética en redes de generación distribuida (Diez, 2012). Por otro lado, en el área de física se han implementado laboratorios de cinemática, conservación de la energía (Henao et al., 2013) y movimiento armónico simple (Contreras-Mendieta et al., 2019).

De la misma manera, en el campo del control automático se han desarrollado varios laboratorios remotos que van desde aplicaciones didácticas a escala hasta entornos industriales completos, para controlar variables físicas como la temperatura, la presión, el flujo y nivel en sistemas de tanques y la velocidad y posición de motores (Domínguez et al., 2005) (Dormido, 2009). También está el desarrollo de un laboratorio en modalidad mixta como LABNET para realizar experimentos de control sobre tres tipos de sistemas físicos: una maqueta de depósito para el control de nivel, una maqueta de control de temperatura, y un sistema de estabilización de barcos, en este caso el laboratorio remoto complementaba las sesiones del laboratorio convencional (Aliane, 2010).

En el diseño e implementación de plataformas y de plantas se integran múltiples herramientas como Matlab y LabVIEW, sin embargo también se suele recurrir a plataformas de código abierto para el desarrollo de las aplicaciones con el fin de evitar el uso de software privativo y con ello reducir los costos del laboratorio (MarCornelio et al., 2019) (de Lima et al., 2016). A su vez, diversos lenguajes de programación como C\# y Java (EJS easy java simulations) se usan con el fin implementarlos en LR avanzados de ingeniería de control y robótica móvil (Fabregas, 2013) y otros como HTML, PHP, JQuery y LDAP para la gestión de reservas con confirmación en el control de acceso para las sesiones de laboratorio (Mazzeo et al., 2016). En cuanto al hardware, para las aplicaciones en instrumentación y control es común el uso de controladores lógicos programables (PLC), también se utilizan tarjetas de desarrollo de bajo costo como Raspberry PI, Arduino y Mbed, especialmente en la construcción de bancos experimentales para adquisición de los datos y su posterior procesamiento (Moreno et al., 2019) (Ortiz et al., 2020).

Finalmente, el estudio exploratorio previo al análisis de la educación en tiempos de COVID-19 desde el objeto de estudio de los laboratorios remotos, evidencia que en tiempos de pandemia y pospandemia los Laboratorios Remoto serán imprescindibles como tecnología aplicada a la educación de las áreas experimentales como ciencias e ingeniería. La Covid-19 aceleró la transformación digital de las organizaciones educativas y está permitiendo que las tecnologías emergentes maduren y consoliden una sinergia estructurada para un mundo visto desde la complejidad de las ciencias. Así mismo, se considera que los trabajos futuros de la educación en ciencias e ingeniería requiere más abordaje técnico, teórico, epistemológico y didáctico, y se determina que la pandemia rescató el trabajo realizado por décadas anteriores en temas de TIC en educación y ha permitido que nuevos investigadores se incorporen al trabajo de la investigación científica en educación desde otras ópticas como la ingeniería y las ciencias aplicadas. 


\section{Metodología}

La investigación fue de enfoque cuantitativo, de tipo Interactivo y transversal (Hernández-Sampieri, 2018), se seleccionó una muestra poblacional conformada por 26 estudiantes del curso de Sistemas de Control de un programa de especialización en Instrumentación y Control Industrial. Para la recolección de datos se utilizaron las técnicas de encuesta y la observación y, como instrumentos se emplearon el cuestionario, la matriz de RAE y la matriz de chequeo de requerimientos. Por su parte, el diseño metodológico se estructuró en cinco fases que se detallan a continuación.

La primera fase consistió en hacer la reflexión sobre la incidencia del COVID-19 en los procesos educativos y la necesidad de apoyar la experimentación con el uso de la tecnología, específicamente, la internet de las cosas (IOT). La segunda fase comprendió un reconocimiento del entorno virtual para la experimentación al cual se le denomina Laboratorio Remoto, en esta fase los estudiantes fueron guiados por el tutor sobre las ventajas del Laboratorio Remoto, sus características y propiedades, a su vez se enfatizó en la telemanipulación y teleoperación ya que, debido a la cuarentena generada por la pandemia, se presentan como una oportunidad para la formación en ciencias e ingeniería al permitir la continuación de los procesos formativos mediante la integración de varios tipos de tecnologías que facilitan la realización de prácticas y experimentos a distancia. Seguidamente, en la tercera fase se organizó el laboratorio remoto de acuerdo a la disponibilidad de los estudiantes puesto que uno de los requerimientos indispensables en la ingeniería de control es la manipulación de las variables intervinientes en las maquinas eléctricas, por ello se configuró el laboratorio de tal forma que cada estudiante desde su casa y sin exposición al contagio por COVID -19 tuviera la oportunidad de controlar el motor eléctrico que se encontraba físicamente en un laboratorio real, con la garantía de manipular las variables del controlador y mientras observaba a través de video en tiempo real el funcionamiento del motor. Luego, en la cuarta fase los estudiantes experimentaron con los diseños realizados para observar la respuesta del motor, finalmente, en la quinta fase se hizo el proceso de reflexión y explicación del comportamiento del sistema de control.

Para el trabajo de campo se diseñó un instrumento con 14 ítems en escala Likert, la fiabilidad se determinó mediante el coeficiente alfa de Cronbach, con un valor de 0.95 indicando un alto grado de confiabilidad.

Respecto a la descripción del laboratorio remoto, se diseñó en torno a una tarjeta de desarrollo ESP32, un sistema en chip (SoC) de bajo costo y con un reducido consumo de energía que fue creado por Espressif Systems, él módulo posee comunicación inalámbrica como Wi-Fi y Bluetooth y un buen paquete de protocolos de comunicación como I2C, SPI, CAN, entre otros, que lo han hecho muy popular en el mundo de la internet de las cosas.

El laboratorio remoto es un sistema de control de velocidad de un motor DC, este motor está conectado a la tarjeta de desarrollo ESP32 en la que se ha implementado un servicio de mensajería a través del protocolo MQTT, uno de los más utilizados en la internet de las cosas debido a que es ligero y simple. Mediante MQTT se envían y reciben los datos desde la página web que está alojada en el servidor de la plataforma Heroku. Por otro lado, el laboratorio remoto posee una cámara web para que el usuario pueda visualizar el funcionamiento del motor. La difusión del video se realiza a través del protocolo WebRTC y mediante una API la página web accede al video en tiempo real.

La página web maneja los servicios por roles, está el de estudiante, profesor y administrador de la plataforma. El estudiante puede reservar el uso del laboratorio en las franjas habilitadas para realizar la práctica, el profesor tiene permitido gestionar la lista de estudiantes y el acceso de cada uno de ellos mediante usuario y contraseña, el administrador tiene control total sobre el laboratorio y puede gestionar la API del video, el control de acceso para los profesores y las demás funciones que tienen los roles de profesor y estudiante. 
Para hacer uso del laboratorio, el estudiante tiene que ingresar con su usuario y contraseña, reservar la hora de trabajo y realizar la práctica en la hora aprobada. Una vez en el laboratorio, puede hacer la adquisición de los datos y el control de velocidad del motor mientras visualiza en tiempo real el funcionamiento de este.

RemoteLAB - Control proporciona el acceso a un sistema estandarizado de control de velocidad de un motor DC para que el estudiante ponga en práctica los conocimientos en el área de sistemas de control. La actividad en el Laboratorio Remoto proporciona al estudiante el afianzamiento de los conocimientos teóricos a través de una práctica de determinación experimental de la función de transferencia a un motor DC y el posterior diseño y comprobación del controlador PID para el mismo. En ella, el estudiante opera remotamente un motor DC, conectado a internet, que está configurado en lazo abierto. Para ello, dispone de una interfaz gráfica de usuario en donde se establece la entrada del sistema y se observa como respuesta la velocidad del motor. Posteriormente, con los datos adquiridos se determina la función de transferencia del sistema usando el método gráfico. Una vez se obtiene la función de transferencia del sistema, el estudiante sintoniza el controlador mediante la variación de los parámetros Kp, Ki, Kd.

A continuación, se enumeran las ventajas que ofrece RemoteLAB - Control

- Operación remota de un motor dc en lazo abierto con la posibilidad de configurar parámetros como tiempo de adquisición de los datos, tiempo de muestreo y voltaje aplicado al motor.

- Visualización de los datos de velocidad del motor y de la entrada aplicada.

- Cálculo de la función de transferencia del sistema Velocidad (RPM) / Voltaje (V), de acuerdo a la gráfica entrada - salida del sistema.

- Posibilidad de descargar los datos adquiridos en formato csv para su tratamiento en software especializado como Matlab.

- Sintonización del controlador PID a través del ingreso de los parámetros $\mathrm{Kp}, \mathrm{Ki}, \mathrm{Kd}$

- Comprobación del controlador diseñado mediante la operación remota y en tiempo real del motor DC.

- Verificación del funcionamiento del sistema a través de video en tiempo real.

- Para una experiencia más significativa, se recomienda el uso del software MATLAB y sus toolboxes de System Identification y SISOTOOL.

La figura 1, presenta la configuración del sistema de laboratorio Remoto y el uso de internet de las cosas para su desarrollo. La figura 2, presenta la interfaz grafica de usuario del laboratorio remoto. 
Figura 1

Configuración del laboratorio remoto y la interconexión con IOT

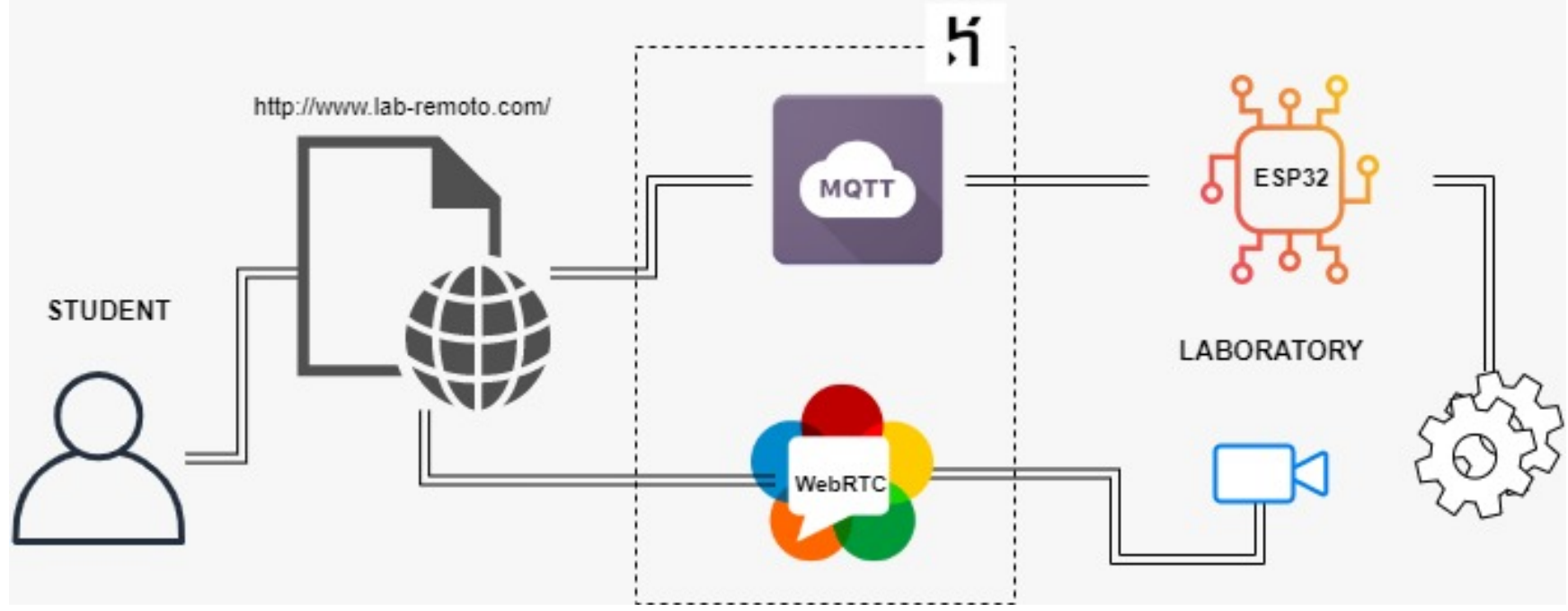

Figura 2

Análisis de las características de laboratorio remoto en el aprendizaje de áreas experimentales.

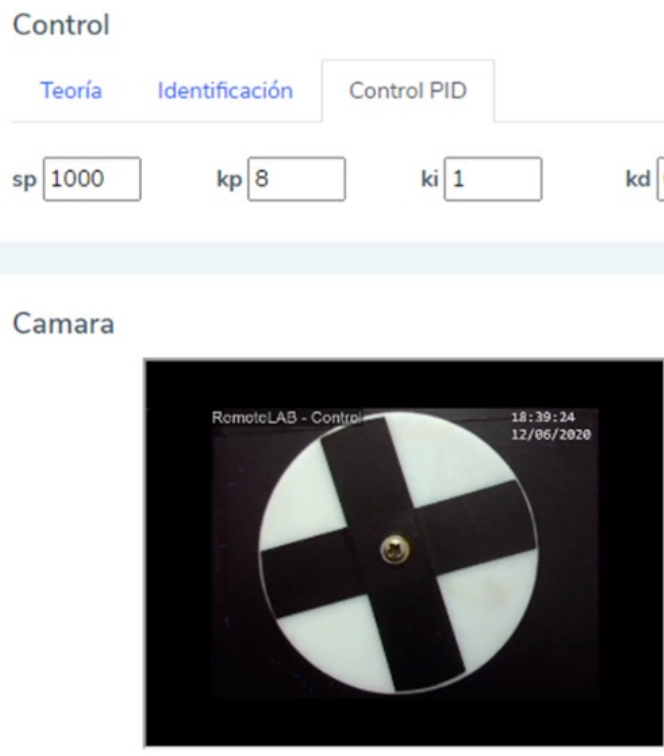

Respuesta del sistema

\section{Resultados}

La concepción de los investigadores sobre la situación posterior a la pandemia por COVID -19 es que la educación en áreas experimentales requiere transformarse y para ello necesita cambios de paradigmas e incorporar tecnologías que permitan una disrupción en los procesos formativos. Las áreas experimentales a nivel de las ciencias y la ingeniería requieren en sus procesos formativos la interacción con laboratorios para la manipulación de variables físicas. Para muchas universidades tradicionales y programas de formación experimental la pandemia los tomó por sorpresa y representó un momento de alta incertidumbre, donde incluso se pensó en cerrar facultades hasta que la emergencia de salud pública finalizara. 
No obstante, las investigaciones en TIC de la década pasada, altamente criticadas por algunos académicos que manifestaban un desinterés por investigaciones en esta área, fueron un punto de partida para que los programas de formación accedieran a iniciar un proceso de enseñanza mediado por la tecnología.

Del avance del área TIC y educación se derivó el tema de transformación digital de las organizaciones, incluidas las organizaciones educativas, también se fortaleció el tema sobre Blended learning o aprendizaje combinado o mixto.

Ante esta panorámica de la educación en tiempos de pandemia, las instituciones, los académicos e investigadores tendrán que generar sinergia para desarrollar la integración de tecnologías que permitan la formación de las áreas experimentales mediadas por tecnologías digitales. Una de las tecnologías digitales emergentes y fortalecidas en la pandemia es la internet de las cosas, esta tecnología es útil para integrarla en el desarrollo de laboratorios remotos que le ofrecen al estudiante una experiencia más enriquecedora que los laboratorios virtuales al trabajar con instrumentos reales pero manipulados a distancia a través de internet.

Desde la perspectiva anterior y en búsqueda de conocer la percepción sobre la pertinencia del uso de laboratorios Remotos e IOT como herramienta educativa para la educación virtual aplicada a la ciencia e ingeniería en tiempos de COVID -19, se realizó un estudio de caso con una población de 26 estudiantes de un programa de especialización en Ingeniería de Control de una universidad colombiana. El estudio analiza la oportunidad que tiene la tecnología de la Internet de las Cosas para el desarrollo de laboratorios remotos de tal forma que sean útiles en la formación de áreas experimentales como la ciencia y la ingeniería, especialmente en programas que son tradicionalmente presenciales y que requieren experimentar y manipular variables físicas. Se propuso el control de la velocidad de un motor de corriente continua utilizado en múltiples artefactos eléctricos. El motor se encontraba en las instalaciones de un laboratorio de automatización industrial y el estudiante, a través de internet, ingresaba los valores de los parámetros del controlador diseñado en la interfaz de usuario de la página web del laboratorio Remoto, al tiempo que observaba a través de una cámara el movimiento del motor y la velocidad que entregaba el equipó de medición dispuesto en el laboratorio. Por tanto, se plantearon tres criterios de percepción, el primer criterio se definió como "el estudiante al ingresar los datos y ver lo cambios de la velocidad en tiempo real tendría la percepción de manipulación del motor como se hace presencial en el laboratorio físico", el segundo criterio fue "la percepción sobre el aporte del laboratorio remoto sobre el aprendizaje seria satisfactorio" y el tercer criterio de percepción planteado fue "el uso de laboratorios remotos es una alternativa viable ante la necesidad de tener programas virtuales o combinados en el área de las ciencias e ingeniería".

La tabla 1, presenta el análisis estadístico de los resultados obtenidos, donde se calculó la media, la desviación estándar, y el valor mínimo y máximo por cada ítem del instrumento. El análisis de los resultados cuantitativos arrojó que el laboratorio remoto es una opción de formación en programas experimentales en tiempos de COVID-19, de acuerdo con el estudio de percepción realizado a los estudiantes objeto de estudio, el laboratorio tiene un grado muy alto de usabilidad, apoya la consolidación del conocimiento, favorece el desarrollo del aprendizaje y permite flexibilidad en los tiempos de ejecución de las actividades. En cuanto al aporte al aprendizaje, los estudiantes manifestaron que el laboratorio remoto permite reforzar el aprendizaje y es pertinente para encontrar la función de transferencia, sintonizar y verificar el diseño del sistema, así mismo, respecto a la viabilidad que brinda los laboratorios remotos para la educación combinada en áreas experimentales manifestaron que el laboratorio remoto presenta en un nivel alto la sensación de estar en un entorno real, presenta en un grado alto la semejanza de la experimentación presencial, genero una satisfacción y facilidad en el proceso de experimentación y finalmente, los estudiantes manifestaron que el laboratorio 
remoto incide en la construcción de un aprendizaje especifico. La figura 3, presenta los resultados obtenidos ante cada característica evaluada.

Tabla 1

Resultados estadísticos de los ítems evaluados

\begin{tabular}{|l|r|r|r|r|r|}
\hline & $\mathrm{N}$ & Media & Desv Std & Mínimo & Máximo \\
\hline Var0001 & 26 & 4,15 &, 88 & 2,00 & 5,00 \\
Var0002 & 26 & 4,12 &, 99 & 1,00 & 5,00 \\
Var0003 & 26 & 3,08 & 1,09 & 1,00 & 5,00 \\
Var0004 & 26 & 3,35 & 1,06 & 1,00 & 5,00 \\
Var0005 & 26 & 3,65 & 1,13 & 1,00 & 5,00 \\
Var0006 & 26 & 4,04 & 1,11 & 1,00 & 5,00 \\
Var0007 & 26 & 2,46 & 1,17 & 1,00 & 5,00 \\
Var0008 & 26 & 3,23 & 1,11 & 1,00 & 5,00 \\
Var0009 & 26 & 4,35 & 1,02 & 1,00 & 5,00 \\
Var0010 & 26 & 3,73 & 1,00 & 1,00 & 5,00 \\
Var0011 & 26 & 3,96 & 1,04 & 1,00 & 5,00 \\
Var0012 & 26 & 3,96 & 1,22 & 1,00 & 5,00 \\
Var0013 & 26 & 4,31 &, 97 & 1,00 & 5,00 \\
Var0014 & 26 & 4,35 &, 94 & 1,00 & 5,00 \\
N Válido (listwise) & 27 & & & & \\
Missing N (listwise) & 1 & & & & \\
\hline
\end{tabular}

Figura 3

Análisis de las características de laboratorio remoto

en el aprendizaje de áreas experimentales

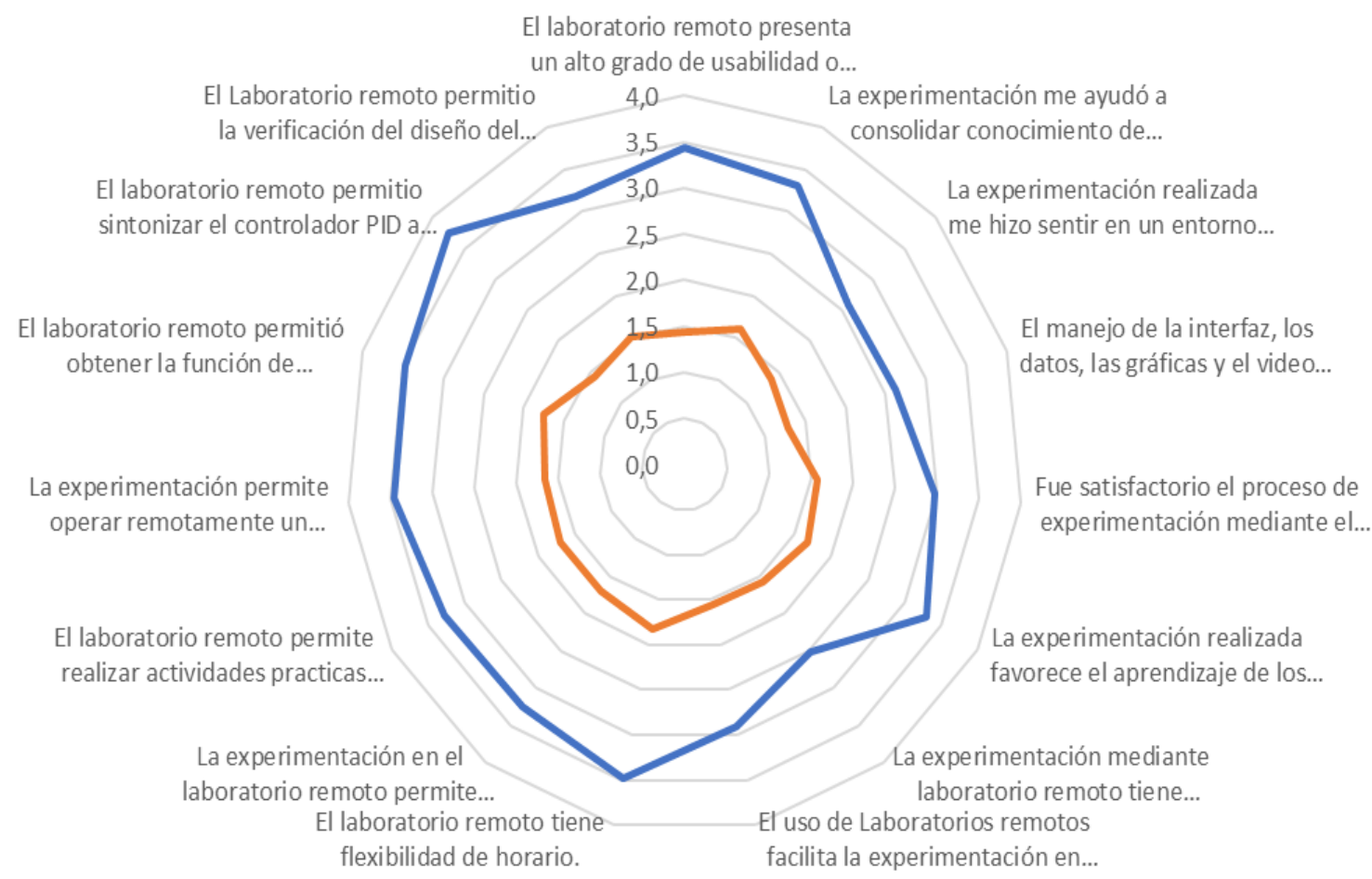




\section{Conclusiones}

El covid-19 generó conflictos en la administración educativa debido a la restricción para continuar con las clases de manera presencial, especialmente en programas con alto contenido práctico experimental como los de las ciencias y las ingenierías. Esos conflictos se fundamentaron en el déficit de tecnologías para el desarrollo de la experimentación científica y técnica que no representaran riesgos de contagio para las personas.

El estudio exploratorio realizado permitió determinar que el uso de laboratorios remotos aporta en el aprendizaje de las ciencias e ingenierías mediante la experimentación del control de variables, equipos, y maquinas reales desde interfaces virtuales, que son una alternativa para implementar en áreas experimentales como las ciencias y la ingeniería que requiere el uso de tecnologías como IOT y desarrollo web y aportan a la construcción de aprendizajes específicos mediante la experimentación con objetos reales manipulados desde una interfaz gráfica web pero con la percepción de la inmersión virtual

Los estudiantes objeto de estudio manifestaron en un grado alto que la experimentación de variables de control mediante la aplicación de técnicas de modelamiento matemático, con el apoyo de una interfaz virtual y con monitoreo en tiempo real a través de video refuerza el aprendizaje de los sistemas de control. También indicaron que el laboratorio remoto no solo da la sensación de experimentación real debido a la realimentación instantánea a través de gráficos y video, sino que cumple con el objetivo propuesto en una práctica sistemas de control, esto es, el laboratorio permite realizar el proceso de identificación para obtener la función de transferencia, realizar el diseño previo, optimizar los parámetros del controlador y, lo más importante, verificar el diseño a través del funcionamiento del equipo físico, todo desde la seguridad de las casas, por tanto de manera general, expresan un alto grado de satisfacción con la experiencia.

\section{Referencias bibliográficas}

Aliane, N. (2010). Experiencia de Uso de un Laboratorio Remoto de Control. Revista Iberoamericana de Automática e Informática Industrial RIAl, 7(1), 85-90. https://doi.org/10.1016/s1697-7912(10)70011-x

Calvo, I., Zulueta, E., Gangoiti, U., López, J. M., Cartwright, H., \& Valentine, K. (2009). Laboratorios remotos y virtuales en enseñanzas técnicas y científicas (Vol. 3, No. 3, pp. 1-21). Ikastorratza.

Canu, M., Duque, M., \& Paris, F.(2015). Laboratorios Remotos: ¿Qué Interés Pedagógico?. Encuentro Internacional de Educación en Ingeniería ACOFI 2015.

Cepal, N. (2020). La educación en tiempos de la pandemia de COVID-19. Comisión Económica para América Latina y el Caribe (CEPAL) y la Oficina Regional de Educación para América Latina y el Caribe de la Organización de las Naciones Unidas para la Educación, la Ciencia y la Cultura. pp.1-20

Contreras-Mendieta, J. A., Sarango-Lapo, C. P., Jara-Roa, D. I., \& Agila-Palacios, M. V. (2019). Implementación de un Laboratorio Remoto (LR), como recurso de apoyo en un sistema de Educación a Distancia. Revista Ibérica de Sistemas e Tecnologias de Informação, (E17), 923-935.

de Lima, J. P. C., Carlos, L. M., Schardosim Simão, J. P., Pereira, J., Mafra, P. M., \& da Silva, J. B. (2016). Design and implementation of a remote lab for teaching programming and robotics. IFAC-PapersOnLine, 49(30), 86-91. https://doi.org/10.1016/j.ifacol.2016.11.133

Diez, D. B. (2012). Laboratorio remoto de eficiencia energética integrado en redes de generación distribuida (Doctoral dissertation, Tesis Doctoral presentada en la Universidad Nacional de Educación a DistanciaEspaña) http://plataforma.quieroauditoriaenergetica.org/CONTADORES/TESIS\%20DOCTORAL\%20\%20LABORATORIO\%20REMOTO\%20EFICIENCIA\%20ENERGETICA.pdf. 
Domínguez, M., Reguera, P., \& Fuertes, J. J. (2005). Laboratorio Remoto para la Enseñanza de la Automática en la Universidad de León (España). Revista Iberoamericana de automática e informática industrial, 2(2), 3645.

Dormido, S., Oyarzún, H. V., Dormido, R., Carralero, N. D., Canto, S. D., García, F. M., ... \& Castro, G. F. (2009). Compartiendo recursos de experimentación a través de Internet: La experiencia automatL@ bs. In La UNED ante el EEES: redes de investigación en innovación docente 2006-2007 (pp. 365-382).

Fabregas, E. (2013). Plataformas Interactivas de Experimentación Virtual y Remota: Aplicaciones de Control y Robótica. Universidad Nacional de Educación a Distancia (UNED). España

Gazzo, M. F. (2020). La educación en tiempos del COVID-19: Nuevas prácticas docentes, ¿̇ nuevos estudiantes? Red Sociales. Revista del Departamento de Ciencias Sociales, 7(2), 58-63.

Henao, J. C., Barrera, J. A. \& Mulcue, L. F. (2013). Physil@ b: conceptos y ejercicios. Universidad Católica de Pereira.

Hernández-Sampieri, R., \& Torres, C. P. M. (2018). Metodología de la investigación (Vol. 4). México^ eD. F DF: McGraw-Hill Interamericana.

Kouzes, R. T., Myers, J. D., \& Wulf, W. A. (1996). Collaboratories: Doing science on the Internet. Computer, 29(8), 40-46.

Loro, F. G. (2018). Evaluación y aprendizaje en laboratorios remotos: Propuesta de un sistema automático de evaluación formativa aplicado al laboratorio remoto VISIR (Doctoral dissertation, UNED. Universidad Nacional de Educación a Distancia (España)).

Luthon, F., \& Larroque, B. (2014). LaboREM-A remote laboratory for game-like training in electronics. IEEE Transactions on learning technologies, 8(3), 311-321.

Mar-Cornelio, O., Santana-Ching, I., \& González-Gulín, J. (2019). Sistema de Laboratorios Remotos para la práctica de Ingeniería de Control. Revista Científica, 3(36), 356-366.

https://doi.org/10.14483/23448350.14893

Masanet, M., Zavalla, E., \& Fernández, A. (2009). Un enfoque integrado para las prácticas de laboratorio en la educación a distancia. Te \& Et, no. 6. http://sedici.unlp.edu.ar/handle/10915/20929

Matarrita, C. A., \& Concari, S. B. (2015). Hacia un estado del arte de los laboratorios remotos en la enseñanza de la física. Revista de Enseñanza de la Física, 27(2), 133-139.

Mazzeo, H. H., Rapallini, J. A., Zabaljauregui, M., \& Rodríguez, O. E. (2016). Diseño de plataforma remota para prácticas de laboratorio Laboratorios virtuales y remotos aplicados a la enseñanza universitaria, $X I X$ Workshop de Investigadores En Ciencias de La Computación (WICC 2017, ITBA, Buenos Aires), 10.

Medina, A. P., Saba, G. H., Silva, J. H., \& de Guevara Durán, E. L. (2011). Los laboratorios virtuales y laboratorios remotos en la enseñanza de la ingeniería. Rev. Educación en Ing, 4, 24-31.

Monaco, L., Vogt, D. M., \& Fransson, T. H. (2013). A New Linear Cascade Test Facility for Use in Engineering Education. Proc. of XXI Biennial Symposium on Measuring Techniques in Turbomachinery, 1-7. http://kth.diva-portal.org/smash/get/diva2:601312/FULLTEXT01.pdf 
Moreira-Segura, C., \& Delgadillo-Espinoza, B. (2015). Virtuality in the educational process: theoretical reflections on its implementation. Revista Tecnología En Marcha, 28(1), 121.

https://doi.org/10.18845/tm.v28i1.2196

Moreno, T. J., López, S. A., Orozco, I. S., \& Balseca, O. F. (2019). Aplicación de un laboratorio remoto para la enseñanza del funcionamiento de componentes oleohidráulicos. Espacios, Vol 40 (39).

Nedic, Z., \& Machotka, J. F. (2007). Remote laboratory NetLab for effective teaching of 1st year engineering students. International Journal of Online and Biomedical Engineering (iJOE), 3(3).

Oliva, H. (2020). La Educación en tiempos de pandemias: visión desde la gestión de la educación superior. Universidad de Guadalajara, 3, 1-16. https://doi.org/10.13140/RG.2.2.27595.54568

Ortiz, S. A. L., Romero, T. J. M., Sampedro, O. F. B., López, E. B. B., \& Pilco, P. E. P. (2020). Diseño y evaluación de un laboratorio remoto para la enseñanza de diseño de circuitos electrohidráulicos. Dominio de las Ciencias, 6(3), 399-424.

Sangrá Morer, A. (2001). Enseñar y aprender en la virtualidad. Educar, 28, 117-131.

Tawfik, M., Sancristobal, E., Martin, S., Gil, R., Diaz, G., Colmenar, A., ... \& Håkansson, L. (2012). Virtual instrument systems in reality (VISIR) for remote wiring and measurement of electronic circuits on breadboard. IEEE Transactions on learning technologies, 6(1), 60-72.

Wulf, W. A. (1989). The national collaboratory-a white paper. Towards a national collaboratory, 17-18.

Zaldívar Colado, A. (2019). Laboratorios reales versus laboratorios virtuales en las carreras de ciencias de la computación. IE Revista de Investigación Educativa de La REDIECH, 10(18), 9.

https://doi.org/10.33010/ie_rie_rediech.v10i18.454

Zamora Musa, R. (2010). Laboratorios remotos: Análisis; características y su desarrollo como alternativa a la práctica en la Facultad de Ingeniería. INGE CUC, 6(1), 281-290.

Esta obra está bajo una Licencia Creative Commons Attribución-NoCommercial 4.0 International

(cc) BY-NC 